\title{
Research on the Application of Digital Image Processing Technology in Intelligent Transportation
}

\author{
Wenshuai $\mathrm{Ji}^{1,}$ a Xiaodong Zhang $^{1, \mathrm{~b}}$ \\ ${ }^{1}$ Henan Mechanical and Electrical Vocational College, Zhengzhou, Henan, China, 451191 \\ ${ }^{\mathrm{a}}$ email, ${ }^{\mathrm{b}}$ email
}

Keywords: Application, Digital Image Processing Technology, Intelligent Transportation

\begin{abstract}
With the development of digital image processing technology, traffic video monitoring technology based on image processing technology has become an important frontier research field of intelligent transportation system. The characteristics of intelligent transportation system and digital image processing technology are briefly introduced. This paper also analyzed emphatically the application of digital image processing technology in intelligent transportation system, such as information collection, license plate recognition, vehicle detection and tracking.
\end{abstract}

\section{Introduction}

With the rapid development of social industrialization and urbanization, the traffic volume of countries all over the world has increased dramatically. How to effectively supervise and control the orderly, high-speed and safe travel of road vehicles has become one of the most important problems that the governments at home and abroad attach great importance to nowadays. However, there are many problems in urban road traffic system, such as congestion, vicious traffic accidents and deteriorating traffic pollution. . Intelligent Transportation System (ITS) is regarded as the basic way to guarantee the safe operation of road traffic. Digital image processing technology has a great theoretical and practical value in the intelligent transportation system.

With the development of economy, urbanization and the acceleration of automobileization, it is required to adopt modern management methods to realize traffic management, which leads to the research of Intelligent Transportation System (ITS). ITS integrates advanced electronic technology, information technology (IT), artificial intelligence (AI), geographic information (GIS), computer technology, communication, sensor technology and system engineering technology into the actual demand of ground transportation, Real-time, accurate and efficient ground transportation system. ITS can improve chaotic traffic conditions, reduce congestion, improve transport efficiency, and improve traffic safety. As an essential part of ITS and the vehicle flow detection system occupies a very important position in ITS. The current domestic and foreign common vehicle flow detection methods are detection coil, TV monitoring, microwave detection, video detection four methods. Among them, the video detection method is superior to other methods more flexible, combined with digital image processing technology, has been widely attention and use.

\section{The Digital Image Processing Technology}

Digital image processing techniques include the use of computers and other electronic devices to complete a series of work, such as image acquisition, acquisition, coding, storage and transmission; image synthesis and generation; image display, rendering and output; image transformation, enhancement, recovery and Reconstruction; image classification, representation and recognition and so on. In addition, image processing technology also includes the hardware and system design and production to achieve the above functions.

Image is the main source of human acquisition and exchange of information, therefore, the application of image processing is bound to involve all aspects of human life and work. With the development of computer video monitoring technology, traffic video monitoring technology based on image processing technology has become an important frontier research field of intelligent transportation system. This article is based on the characteristics of digital image processing, digital 
image processing technology introduced in the intelligent transportation system in all aspects of application.

Digital image processing has three main purposes:

(1) The image gray to do some transformation, to enhance the useful information, inhibit the useless information, so that the image quality improvement in the visual, so that the human eye observation, understanding or computer for further processing. . The main processing techniques are image enhancement, image restoration and image coding.

(2) With a special means to extract, describe and analyze some of the features contained in the image or special information, the main purpose is to facilitate the computer for further analysis and understanding of images, often as pattern recognition, computer vision, etc. deal with. Such image processing techniques include image segmentation, image recognition, feature extraction and the like.

(3) Image data compression, in order to facilitate the storage and transmission of images.

\section{The Overview of Intelligent Transportation System (ITS)}

China's urban transport is facing enormous challenges, and its countermeasures are mainly planning and policy tools, engineering facilities, means of operation and management, as well as the use of high-tech transformation of traditional means of transportation systems, Intelligent Transportation System (ITS-Intelligent Transportation System) An effective way to solve the above contradictions. Intelligent Transportation System (ITS) is at the forefront of transportation science and technology in today's world. It is based on the advanced road facilities, integrating advanced electronic technology, information technology (IT), artificial intelligence (AI), geographic information (GIS) Computer technology, wired / wireless communication (such as ATM, GPS, GPRS, GSM, TETRA), sensor technology and system engineering technology integrated in the practical needs of ground transportation, establish omnidirectional, real-time accurate and efficient ground transportation system; A variety of modes of transport modern, scientific and intelligent management [3]. The emergence of intelligent transportation system is to improve the chaotic traffic conditions, reduce congestion, improve transport efficiency and improve the safety of traffic, traffic environment improvement is in practice with the application was made. Today, the development of ITS is of great significance to the improvement of the environment.

First of all, ITS to the travelers to provide reliable traffic information, at home or anywhere else can use personal travel assistance system to get the information they need in order to help him choose to meet their travel budget and time requirements of traffic Tools and travel routes. The navigation system will guide the driver to reach the destination and the driver will continue to receive the front of the traffic situation information. Drivers and the outside world is fully connected, travel has become easy, safe and comfortable. Digitalization of traffic information is easy to integrate with other service systems. Before entering a city, the driver is informed of the location of the parking lot and how to connect to the bus. He can reserve the parking space. The mobile communication can go directly to the Internet, Sites that access selected services (such as meals, hotels, etc.). In addition, through the exchange of information with the adjacent traffic area, traffic management level will be greatly improved, reducing the lack of information caused by unnecessary delays and wait.

Second, to provide guidance information, a significant reduction in traffic congestion, improve traffic safety. With the aid of the help of artificial intelligence, the control center will integrate the network information continuously monitored, so as to propose the optimal operation scheme of the whole network. In this way, the traffic information of the road network can be monitored. At the same time, the driver receives the guidance information corresponding to the optimized operation plan of the road network. He can choose the driving route according to the guidance information, avoid the crowded road section and select the fast driving route, so as to greatly improve the use of facilities and security of the existing road network foundation. 


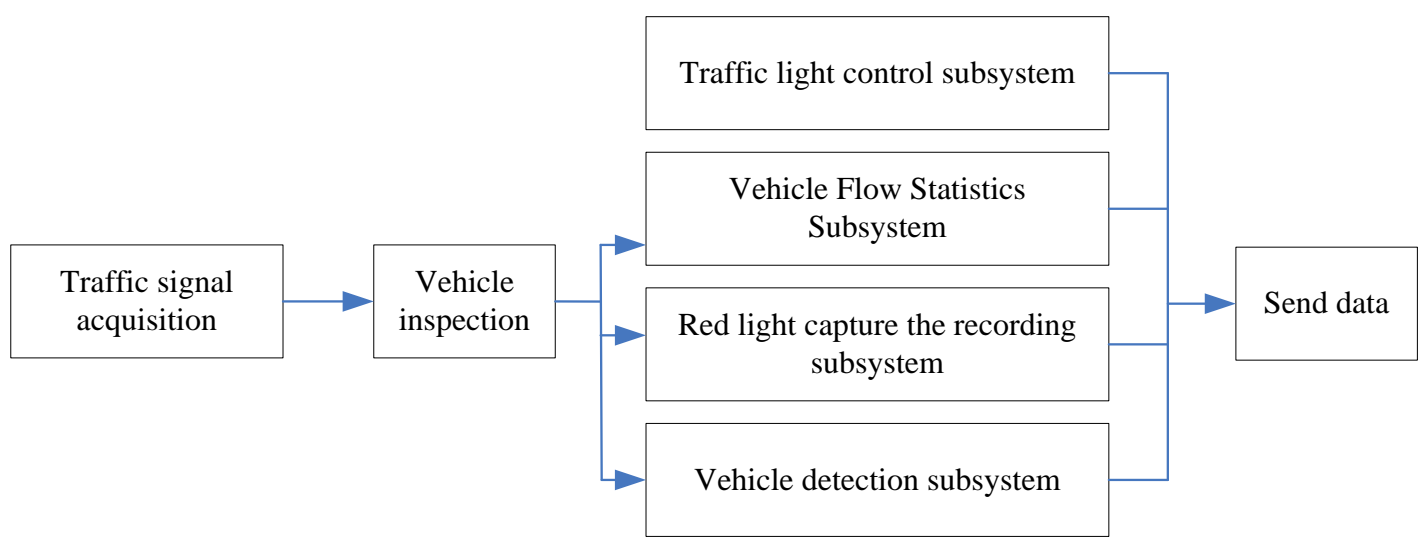

Fig.1 The frame of ITS function

\section{The Application of Digital Image Processing Technology in the Intelligent Transportation}

Traffic Information Collection. Intelligent transportation system can run efficiently, the key depends on obtaining comprehensive, accurate and real-time dynamic traffic information. In the research of intelligent transportation system, it is important to research and develop the traffic information collecting equipment which can effectively obtain the running information on the road, including traffic flow, vehicle speed, vehicle classification, traffic density and so on, to improve the realization of traffic intelligentization. The correct and effective traffic information collection can realize the effective management of the traffic condition and issue the induction information while correctly and timely obtaining the traffic condition information so as to automatically adjust the traffic flow and reduce the time and arrangement for the vehicle to stay in front of the red light when the road is smooth Ease traffic, alarm and so on. Therefore, in the intelligent transportation system, the traffic information gathering technology advancement is the intelligent transport system to be the effective development foundation and enhances the traffic security and the efficiency.

With the rapid development of intelligent traffic collection technology and its analysis technology, traffic information collection has changed from static and manual collection to dynamic and automatic acquisition, from single mode acquisition to multi-mode and multi-method acquisition. Some commonly used traffic information collection methods, such as radar gun, induction coil, GPS velocity measurement, infrared detection, etc., can be a certain degree of vehicle detection, but some of these methods need to slot the road to embed the coil To some extent, damage to the road, affecting road life, some equipment costs are too high, affected by the weather environment and it cannot be comprehensive and efficient collection of information. With the rapid development of digital image processing technology, image analysis method is applied in the field of transportation is more and more widely used. Compared with some traditional traffic information collection methods, it has a comprehensive, efficient, and no interference to the characteristics of the main line of traffic, can truly reflect the traffic flow conditions.

It can extract high quality images from digital images by using computer vision technology, and then obtain the information of traffic characteristics, such as multi-target vehicles and vehicle speed in the range of image. It overcomes the limitation of the traditional method of velocity measurement. But also can accurately locate the space position of the vehicle. In the traffic information collection, no need to control the main line traffic, just to ensure the relevant shooting conditions, but also no observation personnel on-site duties, to a certain extent, reduce the human and material resources to improve collection efficiency.

The License Plate Recognition Technology. In recent years, intelligent transportation system has become the main direction of the current traffic management development. As an important part of intelligent transportation system, the automatic license plate recognition system can greatly improve the efficiency of vehicle management, speed up the automation and intelligentization of traffic management, Automatic Identification System in Parking Lot Charge Management. Closed residential area security management, highway speeding automatic monitoring system and urban 
traffic intersection of the "electronic police" and so has a wide range of applications.

License plate recognition system refers to the technology that can detect the vehicles on the monitored road surface and automatically extract the vehicle license information (including Chinese characters, English letters, Arabic numerals and number plate colors) for processing, mainly by image acquisition. The realization of the system is mainly based on the hardware and software platform, such as digital camera equipment and computer information management system, to collect the vehicle image and make use of advanced image processing. Pattern recognition and artificial intelligence technology, locate the license plate position in the image, extract all the characters that make up the license plate number, and further recognize the characters in the license plate. Letters and numbers, and finally gives the real number of the license plate. Therefore, the license plate recognition system is an important research content of computer vision and pattern recognition technology in the field of intelligent transportation, which is of great significance to modern intelligent transportation.

In practical applications, due to the quality of photos taken by the external factors, such as light intensity. Speed and other factors will affect the quality of photos taken by the vehicle, and because of the weather or speed too fast and other reasons caused the image blurred image acquisition, poor quality, and further affect the follow-up license plate character recognition, so before the image recognition, mainly through the use of digital image processing technology to collect the image pre-processing, including image gray-scale. And then improve the accuracy of subsequent character recognition. In order to improve the processing speed of automatic license plate recognition in the follow-up study of license plate recognition system, we mainly use high-performance computer processing and improving algorithm to improve the speed of image processing and recognition. The complexity of illumination and so on need to be further improved in the future research, and can be seen from the development trend at home and abroad, the intelligent transportation system includes several other aspects such as vehicle type recognition, Vehicle identification, also need further study, and then continue to improve the practicality of license plate recognition.

Motion Vehicle Video Segmentation and Tracking Technology. In the public road traffic management, in order to obtain the vehicle movement data, the early usually mainly used is the induction coil and other hardware measurement method, but this method requires to be set in the road surface, the road is damaged, installation is not convenient, direct The impact of traffic, seriously affect the road life, and if the camera using road video, and then processed by computer software, then greatly increase the vehicle movement data to facilitate the convenience and flexibility.

With the development of computer technology, the road traffic data acquisition no longer needs complex coil equipment, only need to fix the relevant camera in the detection road section, the road taken by the camera is transmitted to the monitoring center through the transmission line (such as optical fiber) The computer of the monitoring center uses the method of digital image processing to calculate the data of road traffic. The effective detection and real-time tracking of moving vehicles in road traffic is the prerequisite for vehicle behavior analysis and recognition, while moving object detection and tracking can be divided into background extraction, moving point extraction, moving point location extraction, moving object tracking Several major steps, through the years of continuous research, for each step of the various algorithms, the researchers have made a comparative analysis of different experimental studies.

\section{Conclusion}

This paper mainly introduces the application of digital image processing technology in intelligent transportation system, such as information collection, license plate recognition, vehicle detection and tracking. Because of the revolutionary development of computer technology and image processing technology, digital image processing technology has become one of the most important technologies in the field of intelligent transportation system. In short, with the continuous development of computer technology, intelligent transportation system based on digital image processing will make greater progress. 


\section{References}

[1] Shi Honglan. Electromechanical Information, Vol. 21 (2011) No 53, p.25-26

[2] Gao Jianping. Chongqing Transportation College, Vol. 1 (2006) No 27, p.74-76

[3] Zhang Zhiqiang. Liaoning Communications Science and Technology, Vol. 05 (2005) No 19, p.144-145

[4] Wang Ran. Business Administration \& Science, Vol. 3 (2016) No 27, p.21-23

[5] Zhu Liling. Information Systems Engineering, Vol. 4 (2016) No 27, p.57-60 